\title{
Pathways from paranoid conviction to distress: exploring the mediator role of Fears of Compassion in a sample of people with psychosis
}

\author{
Maria João Martins, Paula Castilho, Célia Barreto Carvalho, Ana Telma \\ Pereira, Diana Carvalho, Miguel Bajouco, Nuno Madeira, Vitor Santos \& \\ António Macedo
}

To cite this article: Maria João Martins, Paula Castilho, Célia Barreto Carvalho, Ana Telma Pereira, Diana Carvalho, Miguel Bajouco, Nuno Madeira, Vitor Santos \& António Macedo (2017): Pathways from paranoid conviction to distress: exploring the mediator role of Fears of Compassion in a sample of people with psychosis, Psychosis, DOI: 10.1080/17522439.2017.1349830

To link to this article: http://dx.doi.org/10.1080/17522439.2017.1349830

Published online: 19 Jul 2017.

Submit your article to this journal $\llbracket$

View related articles

View Crossmark data $\nearrow$ 


\title{
Pathways from paranoid conviction to distress: exploring the mediator role of Fears of Compassion in a sample of people with psychosis
}

\author{
Maria João Martins a,b (D), Paula Castilho a , Célia Barreto Carvalho ${ }^{a, c}$, Ana Telma Pereirab, \\ Diana Carvalho ${ }^{a}$, Miguel Bajouco ${ }^{b, d}$, Nuno Madeira ${ }^{b, d}$, Vitor Santos ${ }^{b, d}$ and António Macedo ${ }^{b, d}$ \\ aFaculty of Psychology and Educational Sciences, Cognitive and Behavioural Center for Research and Intervention,

 \\ Coimbra, Coimbra, Portugal; 'Faculty of Human and Social Sciences, Department of Psychology, University of Azores, \\ Ponta Delgada, Portugal; ${ }^{\mathrm{d} C o i m b r a}$ University and Hospital Centre, Coimbra, Portugal
}

\begin{abstract}
Fears of Compassion (FOC) relate to experiencing defensive emotions and avoidance reactions when receiving and giving compassion. Three different flows have been identified: giving compassion to others, receiving compassion, and self-compassion. This study sought to explore: FOC within a sample of patients with psychosis; the associations between FOC and paranoia; and the mediator role of FOC in the relationship between paranoid conviction and distress. Seventy-two patients with psychosis (74\% diagnosed with schizophrenia), mostly male (85\%), with a mean age of 33.46 (SD =9.43), were recruited and assessed with measures of paranoia (conviction and distress) and FOC. Participants presented significantly higher levels of FOC than non-clinical samples and lower levels than depressed patients. Different flows of FOC were associated with each other and with paranoia-related measures. A mediation effect of $F O C$ from others and fears of self-compassion was found. Results support the relevance of including FOC in formulation and treatment protocols for psychosis.
\end{abstract}

\section{ARTICLE HISTORY}

Received 31 January 2017

Accepted 28 June 2017

\section{KEYWORDS}

Fears of Compassion; paranoia; conviction; distress; psychosis

\section{Introduction}

Compassion-based intervention models for psychosis suggest the need to activate the safeness-soothing system, a system associated with contented well-being and present moment acceptance (see Gilbert, 2014 for more detailed explanation on affect regulation systems), in addition to reducing the sense of threat (Gumley, Braehler, Laithwaite, Macbeth, \& Gilbert, 2010).

Compassion has been defined as an important output of the soothing system underpinned by caring, helping, and sharing social mentalities (Gilbert, 2014). In individuals with psychosis, self-compassion has been associated with lower levels of positive psychotic symptoms, excitement, and emotional discomfort (Eicher, Davis, \& Lysaker, 2013), negative symptoms and cognitive disorganization (Gumley \& Macbeth, 2014). Bolderston, Newman-Taylor, and Deveson (2014) and Rothwell, Newman Taylor, Bolderston, Deveson, and Maguire (2015) found, with medium to large effect sizes, that cognitive fusion and self-compassion mediated the relationship between paranoia or hallucinations and distress. In a related work, Newman-Taylor and colleagues found that self-compassion predicted general distress (K. Newman-Taylor, personal communication, March 9, 2017). 
Compassion-based interventions for psychosis, aimed at developing more compassionate relationships (with others and self), have been emerging with promising results (e.g. Braehler et al., 2013; Laithwaite et al., 2009). Self-compassion has been preliminarily shown to be relevant to recovery from psychosis (Waite, Knight, \& Lee, 2015). Nevertheless, intervention processes and outcomes may be influenced by problems caused by an overly activated threat-defense system and an underdeveloped soothing system suggested to be present in psychosis (Gumley et al., 2010).

\section{Fears of Compassion (referred as FOC hereinafter) and its relevance for psychosis}

Fear of positive emotions (e.g. affiliative emotions, such as compassion) is developed as a consequence of early aversive experiences when seeking closeness or compassion (Gilbert, McEwan, Catarino, Baião, \& Palmeira, 2014). FOC relate to experiencing defensive emotional responses and a tendency to avoidance reactions when receiving and giving compassion, either to others, from others or self-compassion; and they may constitute major obstacles to recovery (Gilbert, McEwan, Matos, \& Rivis, 2011). The ability for compassionate responding, to other and self, is rooted in and developed by the attachment system. The reactivation of the attachment system (e.g. by compassionate responses), when aversive early experiences have blocked it, might give rise to difficult memories and feelings (Gilbert et al., 2011). Joeng et al. (2017) found that fears of self-compassion and self-compassion serially mediated the relationships between anxious and avoidant attachment and depression and anxiety. FOC have been associated with psychopathological symptoms (e.g. Gilbert et al., 2014).

FOC have the potential to be particularly prevalent in individuals with psychosis due to several aspects. Considering that the origins of FOC are rooted in the attachment system as mentioned above, the fact that people with psychosis often present insecure attachment patterns (Berry, Barrowclough, \& Wearden, 2007) is a relevant indicator of a potential high prevalence of FOC. Attachment styles have been associated with psychotic symptoms and other inter and intrapersonal problems (Gumley, Taylor, Schwannauer, \& MacBeth, 2014). Maladaptative coping strategies tend to emerge in this context as a way to deal with attachment insecurity that might increase psychotic symptoms such as paranoia (e.g. experiential avoidance - Castilho, Martins et al., 2017).

FOC have been reported as most prevalent in people with high levels of shame and selfcriticism (Gilbert et al., 2011) and people with psychosis usually report high levels of both and less selfreassurance abilities (Hutton, Kelly, Lowens, Taylor, \& Tai, 2013). Additionally, previous research has found associations between shame, self-criticism, and paranoid ideation (e.g. Matos, Pinto-Gouveia, \& Gilbert, 2013; Mills, Gilbert, Bellew, McEwan, \& Gale, 2007) and, in samples of people with psychosis, shame was found to be a mediator between paranoia and social safeness (Castilho, Pinto et al., 2017).

Another important competence to be able to feel affiliative emotions is the ability to understand the nature, source, and maintenance of emotions (Gilbert et al., 2012). People with psychosis usually lack emotional awareness and have deficits in emotion-regulation skills (e.g. Lincoln, Hartmann, Köther, \& Moritz, 2015).

In nonclinical samples and samples of patients with depression some of these characteristics (e.g. alexithymia, attachment insecurity, and self-criticism) have been associated with lower levels of compassion, self-compassion and self-reassurance, and fears of positive emotions and compassion, on the other hand (e.g. Gilbert et al., 2012, 2014). In psychosis, it is also theoretically congruent that these characteristics may contribute for FOC. In fact, accounts from clinical studies have stressed the difficulties of people with psychosis in engaging with compassionate-relating (e.g. Mayhew \& Gilbert, 2008).

Authors have stressed the possible reduced therapeutic impact of psychotherapeutic interventions due to the difficulty in experiencing reassurance, compassion and kindness (Gilbert et al., 2011), nevertheless, to date there are no studies exploring the role of FOC in samples with psychosis.

\section{Depression and psychosis}

Within the social rank theory, depression is understood as emerging from a perception of being of an inferior social rank (Gilbert, 1992). On the other hand, paranoia has been conceptualized as a defensive strategy to avoid the activation of negative beliefs about the self (e.g. Bentall, Kinderman, \& Kaney, 1994). Moreover, a specific type of paranoia characterized by higher levels of perceived deservedness 
of persecution - the 'bad me paranoia' (Trower \& Chadwick, 1995) - has been associated with high levels of depression (e.g. Melo, Taylor, \& Bentall, 2006).

Some studies have compared depressive and psychotic symptoms. Results from a nonclinical sample have shown that internalization of aversive experiences would increase vulnerability to depression; and recollections of such experiences would directly and indirectly (through shame) increase paranoia (Pinto-Gouveia, Matos, Castilho, \& Xavier, 2014). Gilbert et al. (2001) explored the similarities between auditory hallucinations and critical thoughts in psychosis and depression and found that similar defensive strategies were activated to respond to both experiences. Hutton et al. (2013) found that people with psychosis engaged more often in hateful self-attacking strategies and used self-reassuring less often than controls, but not when comparing to depressed patients.

FOC have been conceptualized as eliciting defensive strategies to protect the self, therefore, it would be interesting and innovative to explore the differences and similarities between these two populations.

This study sought to characterize FOC in psychosis, comparing it to results from nonclinical and depression samples. We hypothesize that people with psychosis would present higher levels of FOC than nonclinical samples and we want to explore the differences with depression.

We also aimed to explore the role of FOC in the relationship between paranoid conviction, and paranoia-related distress, two important variables when working psychotherapeutically with people with psychosis (considering both the classic cognitive models and the contextual therapies' models of psychosis).

\section{Material and methods}

\section{Participants}

Seventy-two participants were enrolled in this study (see Table 1). Inclusion criteria were: diagnosis of a psychotic-spectrum disorder; age equal or above 18 years old. Patients with cognitive deficits or symptomatology impeding participation (assessed by their psychiatrist) were excluded.

Table 1. Sample's demographic and clinical characteristics.

\begin{tabular}{|c|c|c|c|}
\hline & Male $(n=61)$ & Female $(n=11)$ & Total $(n=72)$ \\
\hline \multicolumn{4}{|l|}{ Age } \\
\hline$M(\mathrm{SD})$ & $33.82(9.84)$ & $31.55(6.85)$ & $33.46(9.43)$ \\
\hline Range & $19-59$ & $25-50$ & $19-59$ \\
\hline \multicolumn{4}{|l|}{ Marital status, $n(\%)$} \\
\hline Single & $55(90.2 \%)$ & $5(45.5 \%)$ & $60(83.3 \%)$ \\
\hline Living with a partner or married & $2(3.3 \%)$ & $5(18.2 \%)$ & $2(2.8 \%)$ \\
\hline Divorced & $3(4.9 \%)$ & $1(9.1 \%)$ & $4(5.6 \%)$ \\
\hline Widower & $1(1.6 \%)$ & 0 & $1(1.4 \%)$ \\
\hline \multicolumn{4}{|l|}{ Occupation, $n$ (\%) } \\
\hline Employed & $20(32.8 \%)$ & $9(81.8 \%)$ & $29(40.3 \%)$ \\
\hline Unemployed & $35(57.4 \%)$ & $1(9.1 \%)$ & $36(50 \%)$ \\
\hline Student & $1(1.6 \%)$ & $1(9.1 \%)$ & $2(2.8 \%)$ \\
\hline Retired & $5(8.2 \%)$ & 0 & $5(6.9 \%)$ \\
\hline \multicolumn{4}{|l|}{ Educational level (years in school) } \\
\hline$M(\mathrm{SD})$ & $10.37(3.48)$ & $12.09(2.55)$ & $10.63(3.39)$ \\
\hline Range & $3-21$ & $8-18$ & $3-21$ \\
\hline \multicolumn{4}{|l|}{ Diagnosis ${ }^{\mathrm{a}}, n(\%)$} \\
\hline Schizophrenia & $48(78.7 \%)$ & $5(45.5 \%)$ & $53(73.6 \%)$ \\
\hline Psychotic disorder NOS & $7(11.5 \%)$ & $1(9.1 \%)$ & $8(11.1 \%)$ \\
\hline Mood disorder with psychotic features & $2(3.2 \%)$ & $2(18.2 \%)$ & $4(5.6 \%)$ \\
\hline Schizophreniform disorder & $1(1.6 \%)$ & $2(18.2 \%)$ & $3(4.2 \%)$ \\
\hline Schizoaffective disorder & $1(1.6 \%)$ & $1(9.1 \%)$ & $2(2.8 \%)$ \\
\hline Substance induced Psychosis & $2(3.3 \%)$ & 0 & $2(2.8 \%)$ \\
\hline \multicolumn{4}{|l|}{ Years at treatment onset } \\
\hline$M(S D)$ & $25.47(7.79)$ & $27.45(4.28)$ & $25.78(7.35)$ \\
\hline Range & $12-59$ & $22-33$ & $12-59$ \\
\hline \multicolumn{4}{|l|}{ Number of hospitalizations } \\
\hline$M(\mathrm{SD})$ & $2.33(1.72)$ & $1.27(.91)$ & $2.17(1.67)$ \\
\hline Range & $0-8$ & $0-3$ & $0-8$ \\
\hline
\end{tabular}

Note: NOS = Not Otherwise Specified.

aDiagnosis following DSM-5 criteria. 


\section{Procedure}

The study was authorized by each recruitment site's Ethics Committee. The sample was collected in five public hospitals and participants were recruited by their psychiatrist. Study's objectives were explained and confidentiality and anonymity ensured. Participants gave informed consent (following the Declaration of Helsinki) and filled in a battery of questionnaires (20-30 min). One researcher was present during the assessment and helped the participants. The researcher also checked for missing data and requested their completion when needed.

\section{Measures}

\section{Paranoia checklist (Freeman et al., 2005)}

An 18-item measure providing a multi-dimension assessment of paranoia, with higher scores indicating greater levels. Comprises three scales: frequency of paranoid thoughts, conviction regarding those thoughts, and distress elicited. The participant is asked to rate each affirmation from 1 (Rarely/Do not believe it/Not distressing) to 5 (At least once a day/Absolutely believe it/Very distressing). Internal consistency was high in the original study and in the Portuguese validation study (Motta, Barreto Carvalho, Pinto-Gouveia, \& Peixoto, 2016). In this study, the subscales 'conviction' and 'distress' were used (good reliability, ranging from .93 to .94 ).

\section{Fears of Compassion Scales (Gilbert et al., 2011)}

The FCS is three scales developed to assess three types of Fears of Compassion. The FOC for others subscale includes 10 items concerning compassion felt for others, related to the sensitivity people have for others' thoughts and feelings (e.g. 'Being too compassionate makes people soft and easy to take advantage of'). The FOC from others subscale refers to the experience of compassion from others as it flows into the self and comprises 13 items (e.g. 'Wanting others to be kind to oneself is a weakness'). The FOC for self includes 15 items on the compassion people have for themselves when mistakes are made or things go wrong (e.g.' I fear that if I am more self-compassionate I will become a weak person'). The participants are asked to indicate their agreement $(0=$ Don't agree at all, $4=$ Completely agree) with higher scores meaning higher levels of FOC. The scores may range from 0 to 40,52 and 60, respectively. In the original study alphas ranged from .78 to .92 and this study from .82 to .90 .

\section{Statistical analysis plan}

Post-hoc power analyses were performed using the G*Power Software (Faul, Erdfelder, Lang, \& Buchner, 2007) and indicated a $99 \%$ and $78 \%$ chance of detecting a large and medium effect size respectively in regression-based analysis. A power of $74 \%$ was found for a Correlation $\rho \mathrm{H} 1=.3$.

Data were analyzed in SPSS version 21 for Windows (IBM Corp., 2012). All variables met the normality assumption using the Kolmogorov-Smirnov test (Lilliefors Significance Correction) and parametric analyses were performed. The magnitude of correlations was analyzed following Cohen's (1977) criteria. To examine indirect effects we used simple mediation models through the Process macro developed by Hayes (2013). We used 95\% bias corrected bootstrap confidence intervals (5000 resampling).

\section{Results}

Results from the mean comparisons of FOC variables are presented in Table 2. All differences were statistically significant with our sample presenting higher levels of FOC comparing to nonclinical samples and lower levels when comparing to patients with depression.

Results from the correlational study are presented in Table 3. FOC were positively and significantly associated with each other and with paranoid conviction and distress. 
Table 2. Means and standard deviations of FOC across studies and mean comparisons.

\begin{tabular}{|c|c|c|c|c|c|c|}
\hline & \multicolumn{2}{|c|}{ FOC for others } & \multicolumn{2}{|c|}{ FOC from others } & \multicolumn{2}{|c|}{ FOC for self } \\
\hline & Mean (SD) & Cohen's $d$ & Mean (SD) & Cohen's $d$ & Mean (SD) & Cohen's $d$ \\
\hline $\begin{array}{l}\text { Present study }(n=72) \text { - } \\
\text { psychosis }\end{array}$ & $23.64(7.24)$ & & $22.89(10.12)$ & & 20.99 (11.96) & \\
\hline $\begin{array}{l}\text { Gilbert et al. }(2011)^{\mathrm{a}} \\
\quad(n=222)-\text { students }\end{array}$ & $21.18(6.71)^{*}$ & .36 & $15.26(7.81)^{*}$ & .91 & $16.12(10.38)^{*}$ & .45 \\
\hline $\begin{array}{l}\text { Gilbert et al. }(2011)^{\mathrm{a}} \\
(n=53) \text { - therapists }\end{array}$ & $10.51(5.51)^{*}$ & 2.02 & $8.81(7.41)^{*}$ & 1.56 & $8.15(6.51)^{*}$ & 1.29 \\
\hline $\begin{array}{l}\text { Gilbert et al. (2012) } \\
\quad(n=185) \text { - students }\end{array}$ & $19.70(7.34)^{*}$ & .54 & $15.26(9.61)^{*}$ & .79 & $14.64(11.74)^{*}$ & .54 \\
\hline $\begin{array}{l}\text { Gilbert et al. (2014) } \\
\quad(n=52) \text { - depression }\end{array}$ & & & $32.6(13.09)^{*}$ & .85 & $39.18(14.34)^{*}$ & 1.41 \\
\hline
\end{tabular}

aThe same study used two different samples.

*Mean different from the mean of the present study at $p<.05$ level.

Table 3. Product moment correlations.

\begin{tabular}{|c|c|c|c|c|c|}
\hline & $M(\mathrm{SD})$ & PC Conviction & PC Distress & FOC for others & FOC from others \\
\hline PC conviction & $47.40(16.38)$ & & & & \\
\hline PC distress & $46.83(17.84)$ & $.60^{* * *}$ & & & \\
\hline FOC for others & $23.64(7.24)$ & $.31^{* *}$ & $.24^{*}$ & & \\
\hline FOC from others & $22.89(10.12)$ & $.25^{*}$ & $.37^{* *}$ & $.47^{* *}$ & \\
\hline FOC for self & 20.99 (11.96) & $.35^{* *}$ & $.44^{* * *}$ & $.38^{* *}$ & $.58^{* *}$ \\
\hline
\end{tabular}

Note: $\mathrm{PC}=$ Paranoia checklist.

${ }^{*} p<.05 ;{ }^{* *} p<.01 ;{ }^{* * *} p<.001$.

Table 4. Total, direct and indirect effects of the simple mediations with Fears of Compassion as mediators (M) in the relationship between paranoid conviction (IV) and paranoia-related distress (DV).

\begin{tabular}{|c|c|c|c|c|c|c|c|c|}
\hline \multirow[b]{2}{*}{ M } & \multirow{2}{*}{$\begin{array}{c}\text { Effect of IV on } \\
M(a)\end{array}$} & \multirow{2}{*}{$\begin{array}{c}\text { Effect of } M \text { on } \\
\text { DV (b) }\end{array}$} & \multirow{2}{*}{$\begin{array}{c}\text { Total effect } \\
\text { (c) }\end{array}$} & \multirow{2}{*}{$\begin{array}{c}\text { Direct effect } \\
\left(c^{\prime}\right)\end{array}$} & \multicolumn{4}{|c|}{ Indirect effect } \\
\hline & & & & & Effect & SE & Lower & Upper \\
\hline FOC for others & $.1361^{* *}$ & .1521 & $6531^{* * *}$ & $6324^{* * *}$ & .0207 & .0352 & -.0361 & .1077 \\
\hline $\begin{array}{l}\text { FOC from } \\
\text { others }\end{array}$ & $.1552^{*}$ & $.4155^{*}$ & $.6531 * * *$ & $.5886^{* * *}$ & .0645 & .0422 & .0071 & .1811 \\
\hline FOC for self & $.2556^{* *}$ & $.3900^{* *}$ & $.6531^{* * *}$ & $.5534^{* * *}$ & .0997 & .0478 & .0333 & .2390 \\
\hline
\end{tabular}

${ }^{*} p<.05 ; *{ }^{* *} p<.01 ; * * * 0<.001$.

Results from the simple mediational analyses regarding FOC subscales as mediators of the relationship between paranoid conviction and distress can be found in Table 4. No mediational effect was found when considering FOC for others as a mediator. Models considering FOC from others and for the self explained $41 \%\left(F=24.1150^{* * *}\right)$ and $42 \%\left(F=24.9164^{* * *}\right)$ of the variance, respectively.

\section{Discussion}

To our knowledge this is the first study to explore the FOC in psychosis and its contribution to the relationship between paranoid conviction and distress. Our results showed that patients with psychosis showed higher levels of FOC comparing with nonclinical samples, but not when comparing with people with depression. Previous research has found higher levels of hated self-attacking in patients with depression when compared to people with psychosis (Hutton et al., 2013), which can contribute to a higher vulnerability to FOC. On the other hand, we hypothesize that some psychotic symptoms (e.g. asociality - a withdrawal from social contact due to indifference or lack of desire) may have influenced these results. This lack of interest in social connection is usually not present in people with depression, which still desire for group inclusion, and this, combined with helplessness, entrapment and defeat feelings (Gilbert \& Allan, 1998), might potentiate FOC. Further research is needed and it would also be 
interesting to explore FOC in subsamples of different types of paranoia since 'bad me' paranoia may resemble depression.

FOC were associated with each other and these results are similar to those found in other clinical samples (Gilbert et al., 2014). However, in contrast with other studies, in our study significant associations were also found of fear of compassion for others with the other types of FOC. We speculate that in people with psychosis the reduced sources of soothing and safeness, especially in social interactions may influence the perception of affiliative emotions as aversive thus creating sources for threat activation, even in the context of expectable soothing experiences. Therefore, people with psychosis may associate the fears of compassionate-relating with each other, in all its different flows, considering their perceived negative consequences (emotional, social, or other) which would function as a common denominator to activate the threat system. Another possible explanation for these results is that this might be influenced by difficulties in social perception, mentalizing and empathic recognition of suffering. These competences are known to be diminished in people with psychosis (Bora, Yucel, \& Pantelis, 2009). Therefore, the inability to recognize and empathically connect with suffering of others/ self might give rise to a fearful response independent of the source of compassion. These are interesting theoretical hypothesis that could be explored in future studies.

Associations between the three FOC and paranoid conviction and paranoid-related distress were significant and a mediating role of FOC (from others and self) was found. These results are in line with previous studies that found self-compassion as a mediator of the relationship of psychosis-type experiences and distress (Bolderston et al., 2014; Rothwell et al., 2015). Other related studies have stressed the importance of lower levels of self-reassurance abilities in persecutory delusions (Hutton et al., 2013), other positive symptoms and insight (Eicher et al., 2013).

Regarding the different pattern of results found for FOC for others, our results are in line with results from previous studies (Gilbert et al., 2011, 2012, 2014) and, as has been suggested, it is possible that different mechanisms are involved in the fears of giving compassion to others (e.g. Gilbert et al., 2014). In psychosis, it may be the case that fears of giving compassion have more relevant role in interference in social-oriented behaviors than with intrapersonal consequences of paranoia (e.g. distress). It has been proposed that, for some individuals from difficult backgrounds, affiliative experiences may activate the threat-defense system, which would block the mentalizing process (Liotti \& Gilbert, 2011). In individuals with psychosis since mentalizing abilities are usually impaired we may hypothesize that a vicious cycle may take place hampering the distress recognition and blocking the motivation to compassionate responding.

Some limitations need to be taken into account. The sample is mostly male and single; therefore, generalization of findings may be difficult. The high prevalence of single participants may indicate a potentially lack of opportunities to receive compassion from and give compassion to others, which might have influence in research and clinical practice. This might stress the need for real-life peerbased supportive interactions that are independent of compassion-based interventions but that can act in a complementary way. The use of self-report measures may have some limitations in assessing delusion-related distress due to low insight. Considering the study's cross-sectional design, causation inferences are not possible. Future longitudinal studies are needed. Due to the current relatively small sample size we were not able to perform other mediation models with higher complexity. Future studies with larger samples could explore a multiple mediation model with the FOC subscales as parallel mediators.

Further study into the FOC mechanisms in psychosis samples seems important not only due to its possible interference in psychotherapeutic processes but also given its possible role in maintaining psychotic symptoms such as paranoia. This study may be integrated in a broader emerging conceptualization of mechanisms of recovery from psychosis, which include enhancing protective mechanisms such as (self-)compassion, acceptance, and mindfulness while buffering the effects of pervasive emotional regulation strategies, such as experiential avoidance or self-criticism.

Notwithstanding its preliminary nature, the present study has important implications for future research and clinical practice. Our results may give insight into possible mechanisms operating between 
paranoid conviction and paranoid-related distress. If replicated these results show the importance of integrating desensitization and exposure in therapy to help patients access or stay with positive emotional states (Gilbert, 2014) before using/inducing such states (e.g. compassion-based exercises) as therapeutic techniques. Strategies of gradual exposure to compassion and affiliative emotions (e.g. to observe compassionate interactions between others in-session and as homework prior more emotionally demanding compassion-based exercises) have been previously recommended (Braehler et al., 2013).

The fact that the FOC for others had the lowest correlation with paranoid conviction and distress and did not have a significant mediator role may indicate that compassion for others might be the safest starting point for training individuals with psychosis in compassion. Although there are no studies on this particular association, the intervention proposed by Braehler et al. (2013) built on this hypothesis by promoting the development of a 'compassionate group mind' to help members of the group.

Considering our results and previous research, including the assessment of FOC in clinical research, clinical practice and future clinical trials with psychosis seems relevant.

\section{Acknowledgements}

The authors would like to acknowledge the collaboration of the patients and clinicians from the Psychiatry and Mental Health Departments of the Baixo Vouga Hospital Centre (Aveiro, Portugal), Coimbra University and Hospital Centre (Coimbra, Portugal), Leiria-Pombal Hospital Centre (Leiria, Portugal), Magalhães Lemos Hospital (Oporto, Portugal) and Tondela-Viseu Hospital Centre (Viseu, Portugal) for which we are very grateful.

\section{Disclosure statement}

The authors declare that they have no conflict of interest.

\section{Funding}

This research has been supported by the first author PhD grant [grant number SFRH/BD/96092/2013], sponsored by FCT (Portuguese Foundation for Science and Technology).

\section{ORCID}

Maria João Martins (iD http://orcid.org/0000-0001-5339-1269

\section{References}

Bentall, R. P., Kinderman, P., \& Kaney, S. (1994). The self, attributional processes and abnormal beliefs: Towards a model of persecutory delusions. Behaviour Research and Therapy, 32, 331-341.

Berry, K., Barrowclough, C., \& Wearden, A. (2007). A review of the role of adult attachment style in psychosis: unexplored issues and questions for further research. Clinical Psychology Review, 27, 458-475. doi:10.1016/j.cpr.2006.09.006

Bolderston, H., Newman-Taylor, K., \& Deveson, C. (2014, December). On decentering from paranoia: Do cognitive fusion and self-compassion mediate the relationship between paranoia and distress?. Presented at ACBS/BABCP ACT/CBS UK and Ireland Conference, Dublin, Ireland.

Bora, E., Yucel, M., \& Pantelis, C. (2009). Theory of mind impairment in schizophrenia: Meta-analysis. Schizophrenia Research, 109(1-3), 1-9. doi:10.1016/j.schres.2008.12.020

Braehler, C., Gumley, A., Harper, J., Wallace, S., Norrie, J., \& Gilbert, P. (2013). Exploring change processes in compassion focused therapy in psychosis: Results of a feasibility randomized controlled trial. British Journal of Clinical Psychology, 52, 199-214. doi:10.1111/bjc.12009

Castilho, P., Martins, M. J., Pinto, A. M., Viegas, R., Carvalho, S., \& Madeira, N. (2017). Understanding the effect of attachment styles in paranoid ideation: The mediator role of experiential avoidance. Journal of Contextual Behavioral Science, 6 , 42-46. doi:10.1016/j.jcbs.2016.11.007

Castilho, P., Pinto, A.M., Viegas, R., Carvalho, S., Madeira, N., \& Martins, M. J. (2017). The mediator effect of external shame in the relationship between paranoia and social safeness in psychosis. Manuscript submitted for publication.

Cohen, J. (1977). Statistical power analysis for the behavioral sciences. New York, NY: Academic Press.

Eicher, A. C., Davis, L. W., \& Lysaker, P. H. (2013). Self-compassion: A novel link with symptoms in schizophrenia? Journal of Nervous and Mental Disorders, 201, 389-393. doi:10.1097/NMD.0b013e31828e10fa 
Faul, F., Erdfelder, E., Lang, A.-G., \& Buchner, A. (2007). G*Power 3: A flexible statistical power analysis program for the social, behavioral, and biomedical sciences. Behavior Research Methods, 39, 175-191. doi:10.3758/BF03193146

Freeman, D., Garety, P., Bebbington, P., Smith, B., Rollinson, R., Fowler, D., .. Dunn, G. (2005). Psychological investigation of the structure of paranoia in a non-clinical population. British Journal of Psychiatry, 186, 427-435. doi:10.1192/bjp.186.5.427

Gilbert, P. (1992). Depression: The evolution of powerlessness. Hove: Lawrence Erlbaum Associates.

Gilbert, P. (2014). The origins and nature of compassion focused therapy. British Journal of Clinical Psychology, 53, 6-41. doi:10.1111/bjc.12043

Gilbert, P., \& Allan, S. (1998). The role of defeat and entrapment (arrested flight) in depression: An exploration of an evolutionary view. Psychological Medicine, 28, 585-598.

Gilbert, P., Birchwood, M., Gilbert, J., Trower, P., Hay, J., Murray, B., ... Miles, J. N. (2001). An exploration of evolved mental mechanisms for dominant and subordinate behaviour in relation to auditory hallucinations in schizophrenia and critical thoughts in depression. Psychological Medicine, 31, 1117-1127. doi:10.1017/S0033291701004093

Gilbert, P., McEwan, K., Catarino, F., Baião, R., \& Palmeira, L. (2014). Fears of happiness and compassion in relationship with depression, alexithymia, and attachment security in a depressed sample. British Journal of Clinical Psychology, 53, 228-244. doi:10.1111/bjc

Gilbert, P., McEwan, K., Gibbons, L., Chotai, S., Duarte, J., \& Matos, M. (2012). Fears of compassion and happiness in relation to alexithymia, mindfulness, and self-criticism. Psychology and Psychotherapy: Theory, Research and Practice, 85, 374-390. doi:10.1111/j.2044-8341.2011.02046.x

Gilbert, P., McEwan, K., Matos, M., \& Rivis, A. (2011). Fears of compassion: Development of three self-report measures. Psychology and Psychotherapy: Theory, Research and Practice, 84, 239-255. doi:10.1348/147608310X526511

Gumley, A., Braehler, C., Laithwaite, H., Macbeth, A., \& Gilbert, P. (2010). A compassion focused model of recovery after psychosis. International Journal of Cognitive Therapy, 3, 186-201. doi:10.1521/ijct.2010.3.2.186

Gumley, A., \& Macbeth, A. (2014). A pilot study exploring compassion in narratives of individuals with psychosis: Implications for an attachment-based understanding of recovery. Mental Health, Religion \& Culture, 17, 794-811. doi:10.1080/1367 4676.2014.922739

Gumley, A. I., Taylor, H. E., Schwannauer, M., \& MacBeth, A. (2014). A systematic review of attachment and psychosis: Measurement, construct validity and outcomes. Acta Psychiatrica Scandinavica, 129, 257-274. doi:10.1111/acps.12172

Hayes, A. F. (2013). Introduction to mediation, moderation, and conditional process analysis: A regression-based approach. New York, NY: Guilford Press.

Hutton, P., Kelly, J., Lowens, I., Taylor, P. J., \& Tai, S. (2013). Self-attacking and self-reassurance in persecutory delusions: A comparison of healthy, depressed and paranoid individuals. Psychiatry Research, 205, 127-136. doi:10.1016/j. psychres.2012.08.010

IBM Corp. 2012. IBM SPSS statistics for windows (version 21.0) [Software]. Armonk, NY: Author.

Joeng, J. R., Turner, S. L., Kim, E. Y., Choid, S. A., Lee, Y. J., \& Kim, J. K. (2017). Insecure attachment and emotional distress: Fear of self-compassion as mediators. Personality and Individual Differences, 112, 6-11. doi:10.1016/j.paid.2017.02.048

Laithwaite, H., O'Hanlon, M., Collins, P., Doyle, P., Abraham, L., Porter, S., \& Gumley, A. (2009). Recovery after psychosis (RAP): A compassion focused programme for individual residing in high security settings. Behavioural and Cognitive Psychotherapy, 37, 511-526. doi:10.1017/S1352465809990233

Lincoln, T. M., Hartmann, M., Köther, U., \& Moritz, S. (2015). Do people with psychosis have specific difficulties regulating emotions? Clinical Psychology and Psychotherapy, 22, 637-646. doi:10.1002/cpp.1923

Liotti, G., \& Gilbert, P. (2011). Mentalizing, motivation and social mentalities: Theoretical consideration and implications for psychotherapy. Psychology and Psychotherapy: Theory, Research and Practice, 84, 9-25. doi:10.1348/147608310X520094

Matos, M., Pinto-Gouveia, J., \& Gilbert, P. (2013). The effect of shame and shame memories on paranoid ideation and social anxiety. Clinical Psychology and Psychotherapy, 20, 334-349. doi:10.1002/cpp.1766

Mayhew, S. L., \& Gilbert, P. (2008). Compassionate mind training with people who hear malevolent voices: A case series report. Clinical Psychology and Psychotherapy, 15, 113-138. doi:10.1002/cpp.566

Melo, S. S., Taylor, J. L., \& Bentall, R. P. (2006). "Poor me” versus “Bad me" paranoia and the instability of persecutory ideation. Psychology and Psychotherapy, 79, 271-287. doi:10.1348/147608305X52856

Mills, A., Gilbert, P., Bellew, R., McEwan, K., \& Gale, C. (2007). Paranoid beliefs and self-criticism in students. Clinical Psychology and Psychotherapy, 14, 358-364. doi:10.1002/cpp.537

Motta, C., Barreto Carvalho, C., Pinto-Gouveia, J., \& Peixoto, E. (2016). Paranoia checklist (Portuguese version): Preliminary studies in a mixed sample of patients and healthy controls. BMC Health Services Research, 16, 81-81. doi:10.1186/s12913016-1423-5

Pinto-Gouveia, J., Matos, M., Castilho, P., \& Xavier, A. (2014). Differences between depression and paranoia: The role of emotional memories, shame and subordination. Clinical Psychology and Psychotherapy, 21, 49-61. doi:10.1002/cpp.1818

Rothwell, T., Newman Taylor, K., Bolderston, H., Deveson, C., \& Maguire, N. (2015, July). On decentering from psychosis: Do cognitive fusion and self-compassion mediate the relationship between psychosis-type experience and distress? Presented at BABCP Annual Conference, Warwick, UK.

Trower, P., \& Chadwick, P. (1995). Pathways to defense of the self: A theory of two types of paranoia. Clinical Psychology: Science and Practice, 2, 263-278. doi:10.1111/j.1468-2850.1995.tb00044.x

Waite, F., Knight, M. T., \& Lee, D. (2015). Self-compassion and self-criticism in recovery in psychosis: An interpretative phenomenological analysis study. Journal of Clinical Psychology, 71, 1201-1217. doi:10.1002/jclp.22211 